\section{Evaluation of Chemotherapy Regimen Management Practice by Oncology- Specialized and Non-specialized Pharmacists Collaboration}

\author{
Yoshitaka Saito, ${ }^{a}$ Kazuki Uchiyama, ${ }^{a}$ \\ Tatsuhiko Sakamoto, ${ }^{a}$ Kosei Kubota, ${ }^{a}$ \\ Hiromitsu Oki, ${ }^{a}$ Miwako Iwai, ${ }^{a}$ Yoh Takekuma, ${ }^{a}$ \\ Yoshito Komatsu, ${ }^{b}$ and Mitsuru Sugawara*,a,c
}

${ }^{a}$ Department of Pharmacy, Hokkaido University Hospital; Kita 14, Nishi 5, Kita-ku, Sapporo 060-8648, Japan: ${ }^{b}$ Cancer Center, Hokkaido University Hospital; Kita 14, Nishi 5, Kita-ku, Sapporo 060-8648, Japan: and ${ }^{c}$ Laboratory of Pharmacokinetics, Faculty of Pharmaceutical Sciences, Hokkaido University; Kita 12, Nishi 6, Kita-ku, Sapporo 060-0812, Japan.

Received October 29, 2020; accepted December 18, 2020

Chemotherapy regimen management is one of the most important oncology pharmacy practices, because chemotherapy is conducted according to the registered regimens. In this study, we evaluated the pharmaceutical practice that assumes the initial confirmation of chemotherapy regimens and the quality of practice sharing between oncology-specialized and non-specialized pharmacists in regimen management committee. Pharmacists initially confirmed the applied regimen prescribed by physicians regarding chemotherapeutic agents and prophylactic supportive care medicines. Following confirmation, the regimens were reviewed by the Hokkaido University Hospital Regimen Management Committee. A total of 233 regimens were reviewed by the committee over three years. In total, 110 pharmaceutical inquiries were conducted, $45 \%$ of inquiries were concerning chemotherapeutic agents, of which approximately half were regarding supportive care medicines. Most inquiries were regarding premedication, followed by those on administration time, solvent of infusion medicines, and dosage. Correction was performed for $84.5 \%$ of inquiries. There was no significant difference in inquiry rates between practice and trial regimens. We have entrusted the first basic regimen review according to the checklist, creation of the chemotherapy plan document, and registry of the adopted regimens in the ordering system from oncology-certified pharmacists to non-certified pharmacists. Basic regimen review was well conducted by a non-certified pharmacist, and a more advanced review was additionally performed by certified pharmacists. In conclusion, we demonstrated the utility of pharmaceutical confirmation in a chemotherapeutic regimen review, suitable review coverage, and quality practice sharing between oncology-certified and non-certified pharmacists, which is one of the recommended methods in chemotherapy regimen review.

Key words chemotherapy regimen management; regimen review; pharmaceutical care; safety management; oncologycertified pharmacist; practice sharing

\section{INTRODUCTION}

Pharmacists assume important and broad roles in the oncology medical team. Pharmacy practice regarding chemotherapy encompasses systematic processes such as the review and management of chemotherapy regimens, supervision and preparation of chemotherapeutic and associated agents, and the development of manuals for safe drug administration, and individual processes such as the evaluation of variety and dose adjustment of medicines, monitoring and managing adverse effects and associated pharmaceutical recommendations, and educating patients. Consequently, advanced knowledge and experience are required for better implementation of this practice. In previous studies, we showed the benefits of successive pharmaceutical care in outpatient chemotherapy. ${ }^{1,2}$ However, the studies were conducted owing to the availability of sufficient management systems for chemotherapy regimens, including prescription ordering systems, as physicians prescribe chemotherapeutic agents using a chemotherapy ordering system. Moreover, as therapeutic and clinical trials require rigorous treatment management, administration using ordering system according to trial protocol is necessary. In contrast, regimen review coverage, such as chemotherapeutic drugs alone or including supportive care medicines, differs in each institution. There are some reports that evaluated regimen review by pharmacists ${ }^{3,4}$; however, detailed information on the utility and most suitable coverage of the review is still unknown.

Within the Hokkaido University Hospital, all infusion chemotherapy regimens, including those for therapeutic trials, are reviewed by the Regimen Management Committee comprising physicians, pharmacists, and nurses. Pharmacists in this committee conduct initial confirmation of regimens, including supportive care medicines, according to the reported phase III trials and/or trial protocols and associated guidelines. This practice is essential for safe chemotherapy management. However, as this practice demands a certain level of professional expertise, oncology-certified pharmacists assume these roles in many institutions. Nevertheless, this may increase the burden of oncology-certified pharmacists, as they have many responsibilities described above. Therefore, we decided to share the practice of basic review and registry of the regimens in the ordering system with non-certified pharmacists from February 2020 (Fig. 1). In this study, we assessed these practices for the enhanced streamlining of operations.

\section{MATERIALS AND METHODS}

Regimen Review Procedure The procedure of the regimen review is shown in Fig. 1. Physicians drafted the basic regimen including dosage, usage, route, interval, and duration of chemotherapeutic agents and prophylactic supportive care medicines. Subsequently, the Japanese Society of Pharmaceutical Health Care and Sciences (JSPHCS)-certified oncology pharmacists or JSPHCS-certified senior oncology pharmacists confirmed these regimens and consulted physicians in case of any issues, and created a chemotherapy plan document containing detailed information about the regimen. Following confirmation, the regimens were reviewed by the Regimen Management Committee. Following approval by this committee, the regimen was registered in the ordering system. We entrusted two tasks from certified pharmacists to non- 


\section{Before sharing}

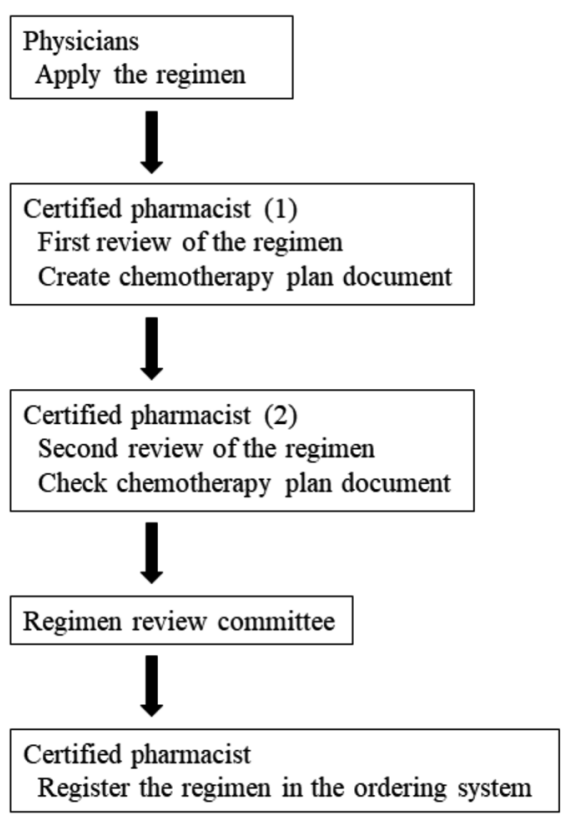

\section{$\underline{\text { After sharing }}$}

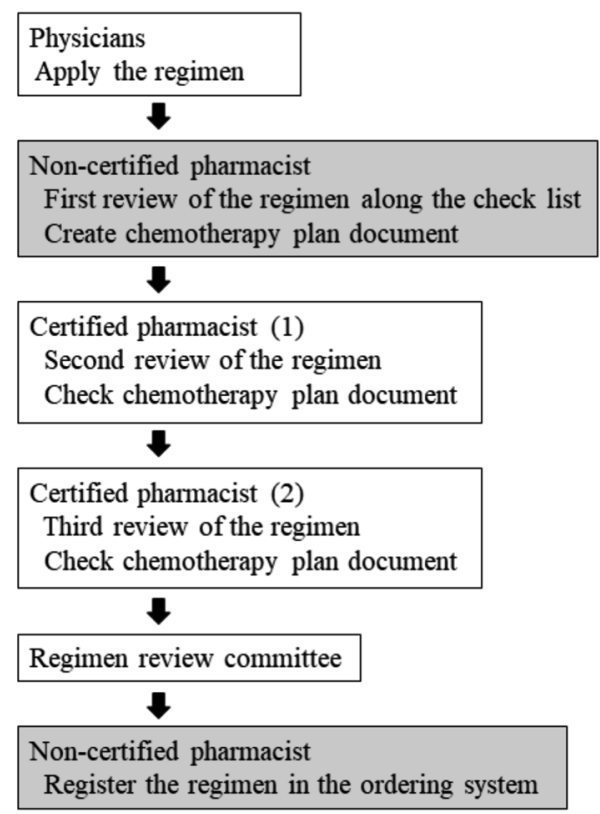

Fig. 1. Change of Pharmacy Practice before and after Practice Sharing

Table 1. Regimen Review Check List

1. I transcribed the applied regimen to chemotherapy plan document.

2. I confirmed the automatic calculation system in the document.

The following should be reviewed using research papers, trial protocols, and product documents.

3. I confirmed the dosage of the medicines in the regimen.

4. I confirmed the dosing schedule of the regimen.

5. I confirmed the dosing interval of the regimen.

6. I confirmed the administration methods of the medicines in the regimen.

7. I confirmed the administration time and infusion rate of the medicines.

8. I confirmed the type and amount of solvent for infusion medicines.

9. I confirmed the criteria for dose reduction or treatment suspension of the chemotherapeutic agents.

10. I confirmed precaution statements such as stability and necessity of light shielding and filter.

11. I confirmed the consistency with existing analogous or similar regimens in all administration methods described above in the regimen.

12. In case of approved medicines, I confirmed the benefits of the drugs.

certified pharmacists: one was the first basic review of applied regimens according to the checklist (Table 1) and creation of the chemotherapy plan document, which are the most timeconsuming tasks, and another was the registry of the adopted regimens in the ordering system.

Evaluation of Data We retrospectively assessed pharmaceutical inquiries regarding the initial confirmation from September 2017 to August 2020 made by the Hokkaido University Hospital Regimen Management Committee. Furthermore, an assessment of practice sharing was conducted by comparing the inquiry rate before and after February 2020. Data on pharmaceutical interventions were extracted from the pharmacy ordering system and e-mails exchanged with the physicians. We considered inquiries according to the checklist to be standard, and defined inquiries that needed additional professional consideration suggested in the guidelines or other relevant documents to be advanced.

Statistical Analysis The rates of regimen inquiry, inquiry adoption, and critical inquiries between practice treatments and clinical and therapeutic trials were assessed using Fisher's exact probability test. The rates of regimen inquiry and inquiry adoption before and after sharing were also evaluated using Fisher's exact probability test. All analyses were performed using JMP version 14.0 statistical software (SAS Institute Japan, Tokyo, Japan). Differences were considered statistically significant when the $p$-value was less than 0.05 .

\section{RESULTS}

Evaluation of Pharmaceutical Review A total of 233 regimens were reviewed by the committee during this period. Details of the reviewed regimens are shown in Table 2. Approximately $50 \%$ of the reviewed regimens concerned practice regimens; of these, approximately $40 \%$ were from therapeutic trials and $9 \%$ were from clinical trials. Details of the pharmaceutical inquiries are shown in Table 3. A total of 110 inquiries (33.9\% of total regimens) were conducted, with $45 \%$ of these inquiries concerning chemotherapeutic agents, 
Table 2. Details of Chemotherapy Regimens Assessed by the Regimen Management Committee

\begin{tabular}{lr}
\hline \hline Number of the committee meeting held & 36 \\
Total regimens & 233 \\
Practice regimens & 123 \\
Therapeutic trials & 89 \\
Clinical trials & 21 \\
Category of treatment regimens & \\
Gastroenterological cancer & 51 \\
Hematological cancer & 48 \\
Lung cancer & 20 \\
Pediatric cancer & 20 \\
Urological cancer & 20 \\
Head and neck cancer & 16 \\
Gynecological cancer & 14 \\
Breast cancer & 11 \\
Brain tumor & 8 \\
Cutaneous cancer & 6 \\
Others & 19 \\
\hline
\end{tabular}

Table 3. Details of Inquiries by Pharmacists

\begin{tabular}{lr}
\hline \hline Number of inquiries & 110 \\
About chemotherapeutic agents & 49 \\
About supportive care medicines & 54 \\
Others & 7 \\
Number of regimens conducted the inquiries & 79 \\
About chemotherapeutic agents & 36 \\
About supportive care medicines & 42 \\
Both & 1 \\
Inquiries related to & 32 \\
Premedication & 17 \\
Administration time & 15 \\
Solvent of infusion medicines & 11 \\
Dosage & 11 \\
Other supportive care medicines & 7 \\
Administration interval & 6 \\
Addition or deletion of associated regimens & 11 \\
Others & 93 \\
Number of corrections by the inquiries & 38 \\
Corrections about chemotherapeutic agents & 50 \\
Corrections about supportive care medicines & 51 \\
Others & 43 \\
Number of corrected regimens & 46 \\
Regimens corrected about chemotherapeutic agents & \\
Recommendation of administration methods or update & 46 \\
Number of regimens conducted recommendations & 31 \\
\hline
\end{tabular}

of which approximately half were regarding supportive care medicines. Most inquiries concerned premedication (32 out of $110,29.1 \%$ ), followed by administration time (17 out of 110 , $15.5 \%$ ), solvent of infusion medicines (15 out of $110,13.6 \%$ ), dosage (11 out of $110,10.0 \%$ ), other supportive-care medicines (11 out of $110,10.0 \%$ ), administration interval ( 7 out of 110 , $6.4 \%$ ), and addition or deletion of associated regimens (6 out of $110,5.5 \%$ ). Corrections were performed for $84.5 \%$ (93 out of 110) of the inquiries, and approximately 30\% (71 out of 233) of the applied regimens required modifications. In particular, pharmaceutical recommendations regarding administration methods were provided for $18.5 \%$ (43 out of 233 ) of the applied regimens, and all were adopted.

We also assessed the differences in inquiry and adoption rate between practice regimens and therapeutic and clinical trials. Inquiry and adoption rate tended to be higher in practice regimens than in trials, but the difference was not significant. However, the rate of critical inquiries such as dosage, administration time, and dose interval of chemotherapeutic agents, which are directly linked to agent efficacy and adverse effects, did not differ between the regimens (Table 4).

Evaluation of Practice Sharing The difference in inquiry rate before and after practice sharing is shown in Fig. 2. We reviewed 188 regimens before sharing (from September 2017 to January 2020) and 45 regimens after sharing (from February 2020 to August 2020). The inquiry rate was $31.9 \%$ (60 out of 188 regimens) before and $42.2 \%$ (19 out of 45 regimens) after practice sharing, without a statistically significant difference. The adoption rate was $83.1 \%$ (69 out of 83 inquiries) before practice sharing and $88.9 \%$ (24 out of 27 inquiries) after practice sharing, with no statistical difference $(p=0.35)$. Approximately $80 \%$ (21 out of 27 inquiries) of the inquiries were conducted by non-certified pharmacists according to the checklist, and only more advanced inquiries were conducted by certified pharmacists.

\section{DISCUSSION}

As chemotherapy is associated with potent and broad adverse effects, risk management of treatments is the most important role of oncology pharmacists. Therefore, strict management of its administration is required. The utility of face-to-face pharmaceutical care and agreements, such as collaborative drug therapy management or protocol-based pharmacotherapy management, has been reported. ${ }^{1,2,5-12)}$ Such measures ultimately contribute to safe and less onerous chemotherapy implementation. Consequently, these important pharmacy practices are a welcome addition to established practices within the clinical oncology setting. However, such additional practices have been developed owing to their re-

Table 4. Inquiry and Adoption Ratio between Practice Regimens and Trial Regimens

\begin{tabular}{lccc}
\hline \hline & Practice regimens & Trial regimens & $p$-Value \\
\hline Number of the regimens & 123 & 110 & - \\
Inquired regimens $(n, \%)$ & $49(39.8 \%)$ & $30(27.3 \%)$ & 0.05 \\
Number of total inquiries & 62 & 48 & - \\
Adoption of inquiry $(n, \%)$ & $56(90.3 \%)$ & $37(77.1 \%)$ & 0.07 \\
Regimens with critical inquiry $(n, \%)$ & $4(3.3 \%)$ & $7(6.4 \%)$ & 0.36 \\
\hline
\end{tabular}

Trial regimens include therapeutic and clinical trials. 


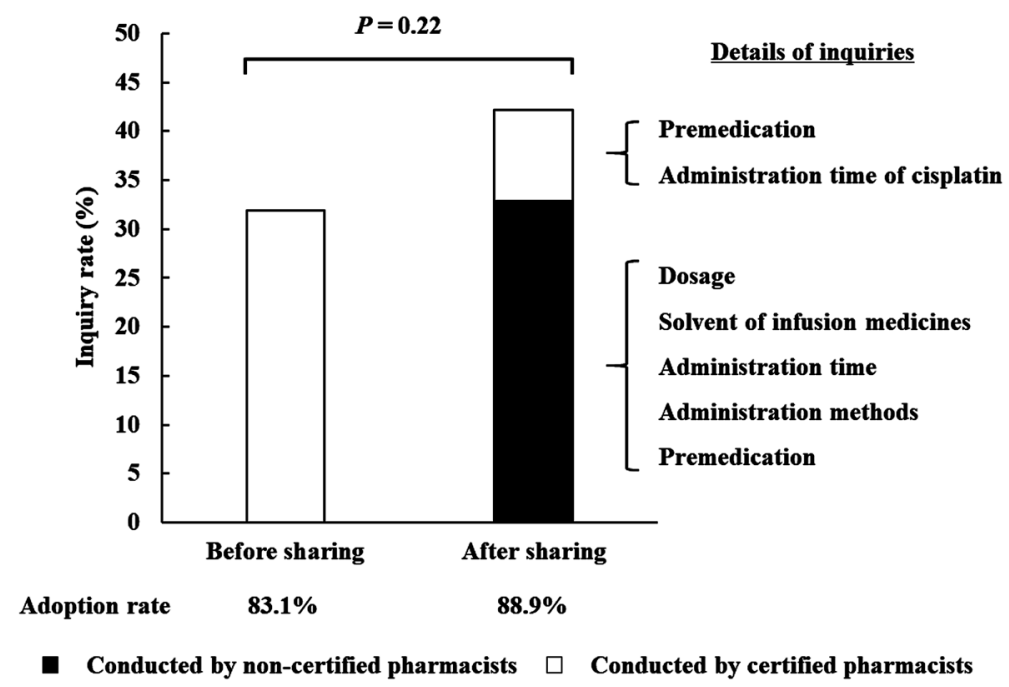

Fig. 2. Evaluation of Inquiry and Adoption Rate before and after Practice Sharing

cords in well-ordered and precise management systems. In this study, we assessed the pharmaceutical review system involving oncology-specialized pharmacists in the regimen management committee.

The results revealed that approximately $30 \%$ of the applied regimens required modifications, which was similar to the findings of a previous report. ${ }^{3)}$ In particular, critical inquiries regarding chemotherapeutic agents such as dosage, administration time, and dose interval were noted in $4.7 \%$ of the applications, suggesting that the double-check system was indeed effective. As expected, the ratio of regimen inquiry and their adoption was higher in practice regimens than in trials. This is because the administration protocol is more strictly provided in therapeutic and clinical trials. In contrast, the critical inquiry rates were similar between practice regimens and trials. Therefore, we propose that the review of trial regimens as well as practice regimens is necessary.

In addition, 54 inquiries regarding supportive care medicines were conducted for 43 regimens (18.5\%). Such treatments are also important for treatment continuation along with safety management and preservation of patient QOL. These should be reviewed according to the latest guidelines, such as those for antiemetics, ${ }^{13)}$ fever and neutropenia, ${ }^{14)}$ and renal injury. ${ }^{15)}$ Moreover, pharmaceutical recommendations, mainly concerning supportive care medicines, were made in approximately $20 \%$ of the applied regimens, and all were adopted. Consequently, we strongly recommend that the committee also reviews prophylactic supportive care medicines in the regimens to avoid confusion and risk of iatrogenic errors, and pharmacists should assume this responsibility.

The above results demonstrated that the suitable coverage of regimen review includes all practice and trial regimens as well as chemotherapeutic agents and prophylactic supportive care medicines. Review of all matters described above enables physicians to provide standardized and certified chemotherapy to patients. If there is a necessity to modify the regimen according to changes in patient condition, pharmacists should be consulted for confirmation. Furthermore, regimens, particularly regarding supportive care medicines, should be updated according to the latest guidelines and reports.

We also assessed pharmacy practice sharing in the regimen review. The rate of regimen inquiry and inquiry adoption were similar before and after sharing. The details of inquiries found by non-certified pharmacists were dosage, solvent of infusion medicines, administration time, administration methods of the infusion medicines, which were described in the checklist, and premedication, accounting for approximately $80 \%$ of the inquiries. Inquiries conducted by certified pharmacists were more advanced, such as premedication selection or administration time of cisplatin, which application was $30 \mathrm{~min}$ in therapeutic trial and suggested to change to $60 \mathrm{~min}$ as well as clinical practice in Japan. In particular, as all standard assessments of the applied regimens according to the checklist were conducted in the first review and no additional standard inquiries were performed in the second or third review by certified pharmacists, it can be suggested that a basic review using the checklist by non-certified pharmacists was well-conducted; however, a more advanced review should be additionally performed by certified pharmacists. As inquiry and adoption rates before and after sharing were not different, the level of the reviews was similar. Consequently, the burden on certified pharmacists was reduced as the most time-consuming tasks were shared with non-certified pharmacists.

It is also important for certified pharmacists to educate non-certified pharmacists, especially junior pharmacists; thus, application of the described chemotherapy-regimen management practice may provide good opportunities to learn and experience as first regimen review requires the ability of paper-reading proficiency, evaluation of applied data, and responsibility. Moreover, it is desirable to increase the number of representatives who can conduct the practice rather than depend on any individual's ability to ensure continuity in the quality of clinical practice and education.

In fact, reviewing time increased in the initial review by sharing, because certified pharmacists were more accustomed to the practice than non-certified ones (data not shown). However, there was no difference in the quality and labor time of regimen registration in the ordering system (data not shown). This is an evolving process, and we need to consider shortening the reviewing practice time. Accordingly, we will update the sharing practice.

There are a few limitations to this study. First, this was a retrospective study conducted at a single institution. Second, as the initial review was conducted by oncology-specialized 
pharmacists, the results obtained could differ from those obtained from non-specialized pharmacists alone.

In conclusion, we demonstrated the utility of pharmaceutical confirmation in a chemotherapeutic regimen review, suitable review coverage, and quality practice sharing between oncology-certified and non-certified pharmacists. The results obtained in this study reveal the emerging importance of pharmacy practice in chemotherapy management and will aid in the construction of more certified and streamlined pharmacy practices for the overall benefit of patients with cancer.

Conflict of Interest YS, KU, TS, KK, HO, MI, YT, and MS have no conflicts of interest. YK reports honoraria from Pfizer, Novartis, and Bayer; research funding from Eli Lilly, MSD, Ono Pharmaceutical, Novartis, Bayer, Chugai Pharma, Yakult, and Taiho; and provided speaker services for Eli Lilly, Chugai Pharma, Merck Serono, Novartis, Pfizer, Bayer, and Taiho.

\section{REFERENCES}

1) Saito $Y$, Harada S, Kobayashi M, Ueda K, Yamazaki K, Kumai M, Miyamoto T, Kasashi K, Yamada T, Komatsu Y, Iseki K. Survey of the efficacy of long-term and successive pharmaceutical care in outpatient chemotherapy by oncology pharmacy specialists. Yakugaku Zasshi, 138, 1409-1416 (2018).

2) Saito Y, Uchiyama K, Sakamoto T, Yamazaki K, Kubota K, Takekuma Y, Komatsu Y, Sugawara M. Pharmaceutical care contributes to the advanced management of patients receiving immune checkpoint inhibitors. Biol. Pharm. Bull., 43, 1969-1974 (2020).

3) Hoshi I, Watanabe H, Nakai Y, Yamasaki K, Kameyama M. Efforts of pharmacists in regimen management for safe cancer chemotherapy. Gan To Kagaku Ryoho, 37, 363-367 (2010).

4) Diaz AH, Bubalo JS. Evaluation and implementation of chemotherapy regimen validation in an electronic health record. J. Oncol. Pharm. Pract., 20, 461-468 (2014).

5) Muluneh B, Schneider M, Faso A, Amerine L, Daniels R, Crisp B, Valgus J, Savage S. Improved adherence rates and clinical outcomes of an integrated, closed-loop, pharmacist-led oral chemotherapy management program. J Oncol. Pract., 14, e324-e334 (2018).

6) Colombo LRP, Aguiar PM, Lima TM, Storpirtis S. The effects of pharmacist interventions on adult outpatients with cancer: a systematic review. J. Clin. Pharm. Ther., 42, 414-424 (2017).
7) Le S, Chang B, Pham A, Chan A. Impact of pharmacist-managed immune checkpoint inhibitor toxicities. J. Oncol. Pharm. Pract., 1078155220928407; Epub ahead of print (2020). doi: $10.1177 / 1078155220928407$

8) Kimura M, Go M, Iwai M, Usami E, Teramachi H, Yoshimura T. Evaluation of the role and usefulness of a pharmacist outpatient service for patients undergoing monotherapy with oral anti-cancer agents. J. Oncol. Pharm. Pract., 23, 413-421 (2017).

9) Suzuki H, Suzuki S, Kamata H, Sugama Y, Demachi K, Ikegawa K, Igarashi T, Yamaguchi M. Impact of pharmacy collaborating services in an outpatient clinic on improving adverse drug reactions in outpatient cancer chemotherapy. J. Oncol. Pharm. Pract., 25, $1558-1563$ (2019)

10) Ikesue H, Kusuda K, Satsuma Y, Nishiwaki F, Miura R, Masuda Y, Hirabatake M, Muroi N, Fujimoto D, Morimoto T, Tomii K, Hashida T. Evaluation of the usefulness of protocol-based pharmacistfacilitated laboratory monitoring to ensure the safety of immune checkpoint inhibitors in patients with lung cancer. J. Clin. Pharm. Ther., 45, 1288-1294 (2020).

11) Merten JA, Shapiro JF, Gulbis AM, Rao KV, Bubalo J, Lanum S, Engemann AM, Shayani S, Williams C, Leather H, WalshChocolaad T. Utilization of collaborative practice agreements between physicians and pharmacists as a mechanism to increase capacity to care for hematopoietic stem cell transplant recipients. Biol. Blood Marrow Transplant., 19, 509-518 (2013).

12) Hansen EA, Pietkiewicz JM, Blum BL. Evaluation of the feasibility and utility of a pharmacist-centered collaborative drug therapy management program for oncology-based symptom management. J. Pharm. Pract., 29, 206-211 (2016).

13) Hesketh PJ, Kris MG, Basch E, Bohlke K, Barbour SY, Clark-Snow RA, Danso MA, Dennis K, Dupuis LL, Dusetzina SB, Eng C, Feyer PC, Jordan K, Noonan K, Sparacio D, Lyman GH. Antiemetics: ASCO guideline update. J. Clin. Oncol., 38, 2782-2797 (2020).

14) Taplitz RA, Kennedy EB, Bow EJ, Crews J, Gleason C, Hawley DK, Langston AA, Nastoupil LJ, Rajotte M, Rolston K, Strasfeld L, Flowers CR. Outpatient management of fever and neutropenia in adults treated for malignancy: American Society of Clinical Oncology and Infectious Diseases Society of America Clinical Practice Guideline Update. J. Clin. Oncol., 36, 1443-1453 (2018).

15) Horie S, Oya M, Nangaku M, Yasuda Y, Komatsu Y, Yanagita M, Kitagawa Y, Kuwano H, Nishiyama H, Ishioka C, Takaishi H, Shimodaira H, Mogi A, Ando Y, Matsumoto K, Kadowaki D, Muto S. Guidelines for treatment of renal injury during cancer chemotherapy 2016. Clin. Exp. Nephrol., 22, 210-244 (2018). 